\title{
A Nonlocal Contour Dynamics Model for Chemical Front Motion
}

\author{
Dean M. Petrich ${ }^{*}$ and Raymond E. Goldstein \\ Department of Physics, Joseph Henry Laboratories \\ Princeton University, Princeton, NJ 08544
}

\begin{abstract}
Pattern formation exhibited by a two-dimensional reaction-diffusion system in the fast inhibitor limit is considered from the point of view of interface motion. A dissipative nonlocal equation of motion for the boundary between high and low concentrations of the slow species is derived heuristically. Under these dynamics, a compact domain of high concentration may develop into a space-filling labyrinthine structure in which nearby fronts repel. Similar patterns have been observed recently by Lee, McCormick, Ouyang, and Swinney in a reacting chemical system.
\end{abstract}

*dpetrich@puhep1.princeton.edu

${ }^{\dagger}$ gold@puhep1.princeton.edu 
In the study of chemical systems with both reactions and diffusion, one may discern two broad classes of spatial patterns: extended and compact. Extended patterns typically arise from supercritical symmetry-breaking bifurcations [1]. In the two dimensional case, which we consider here, they are often regular, periodic structures such as arrays of stripes, discs, or hexagons [2]. Compact patterns, or localized states, appear in systems with subcritical bifurcations via a nucleation process [3], and typically take the form of a single one of the repeating units found in extended systems. Both classes of patterns are often described in terms of a competition between two chemical species: an autocatalytic "activator" and its "inhibitor."

Localized states can exhibit a fingering instability [㑑]. One can imagine that these fingers may grow and branch until a complicated labyrinthine pattern fills the entire plane. Such structures may actually be metastable states; barriers to domain fission may prevent the pattern from evolving into the ground state (presumably a regular array of stripes) from an initial condition which is topologically different. Qualitatively similar kinds of pattern formation appear in other systems [5].

Complicated, labyrinthine pattern evolution in a chemical system has recently been observed by Lee, McCormick, Ouyang, and Swinney [6] in an iodate-ferrocyanide-sulfite reaction (see Fig. 1). The patterns are composed of regions with one of two different chemical compositions. This system appears to be bistable; if it is prepared with one of the two possible uniform compositions, it will persist in that state. Nontrivial pattern formation requires a nucleation site in an otherwise uniform background. These experiments also indicate that in the formation of patterns, nearby boundaries or fronts repel each other. We will use these results as a guide to the general features one would like to see in a model system [7].

It is natural to seek a representation of the dynamics of these and similar patterns in terms of the interface between domains of different composition; in order to capture the properties of repulsion and nonintersection, such a contour dynamics must be nonlocal, coupling segments of the interface that are distant in arclength yet close in space. The purpose of this letter is twofold: to give an intuitive construction of a fully nonlocal curve 
dynamics from a set of reaction-diffusion equations and to show that it is useful for describing pattern formation. The evolution equation is written in terms of the intrinsic geometry of the curve, and is valid for pattern evolution far beyond the linear instability of localized states. In this simplified form, it is easier to identify the physics responsible for the destabilization of a compact pattern, the tendency of a pattern to grow or shrink, and the interaction between different portions of the interface. The curve dynamics is studied numerically and is shown to reproduce the qualitative features of the experimental patterns.

The reaction-diffusion pair studied here is similar to models of spiral wave formation [8] and nerve impulse propagation [9]. With $u$ the activator and $v$ the inhibitor, we consider

$$
\begin{gathered}
u_{t}=D \nabla^{2} u-\mathrm{F}^{\prime}(u)-\rho v, \\
\epsilon v_{t}=\nabla^{2} v-\alpha v+\beta u .
\end{gathered}
$$

The nonlinear function $\mathrm{F}(u)$, with derivative $\mathrm{F}^{\prime}$, embodies the autocatalytic nature of the activator. It is typically a polynomial in $u$ with a double well structure whose minima, not necessarily of equal depth, we label $u_{ \pm}$. Patterns like that in Fig. 1 can then form in which regions of $u \simeq u_{+}$(e.g. white) are surrounded by a region of $u \simeq u_{-}$(black). Inhibition of $u$ is achieved for $\rho>0$, while the couplings $\alpha$ and $\beta$ reflect the self-limiting behavior of the inhibitor and its stimulation by the activator, respectively. The small number $\epsilon$ defines the fast-inhibitor limit. This limit is opposite to the limit assumed in phase-field models [10] and spiral wave dynamics [8]. The fast inhibitor assumption appears to be the simplest assumption that allows the elimination of one of the fields, giving rise to spatial nonlocality for the remaining field without introducing temporal nonlocality. A similar calculation can be done for the slow-inhibitor limit [11], but due to temporal nonlocality, the resulting equations are more complicated, obscuring the essential physics.

We begin by discussing the interaction of fronts, illustrating the property of self-avoidance present in the reaction-diffusion pair (1), for certain parameter ranges. Figure 2 shows a simulation of a one-dimensional version of (1) with periodic boundary conditions. The 
patterns are defined by the sharp interfaces of the $u$ field. The parameters are such that the $u \simeq u_{+}$region expands into the $u \simeq u_{-}$region. When the interfaces get too close, the exponential tails of the $v$ field begin to overlap, causing a repulsion which stabilizes the final field configuration. In the derivation of the two-dimensional contour dynamics, we shall analytically see the source of this repulsion.

To derive an interface evolution equation for two-dimensional patterns, we take the fastinhibitor limit, setting $\epsilon=0$ and thus slaving $v$ to $u$,

$$
v(\mathbf{x}, t)=\beta \int d^{2} x^{\prime} \mathcal{G}\left(\left|\mathbf{x}-\mathbf{x}^{\prime}\right|\right) u\left(\mathbf{x}^{\prime}\right)
$$

where the Green's function $\mathcal{G}(r)=\mathrm{K}_{0}(r / \xi) /(2 \pi)$, and $\xi=\alpha^{-1 / 2}$. Substituting for $v$ in equations (1a), we obtain the variational dynamics $u_{t}=-\delta \mathcal{F}[u] / \delta u$, with

$$
\begin{aligned}
\mathcal{F}[u]= & \int d^{2} x\left\{\frac{1}{2} D(\nabla u)^{2}+F(u)\right\} \\
& +\frac{\rho \beta}{2} \int d^{2} x \int d^{2} x^{\prime} u(\mathbf{x}) \mathcal{G}\left(\left|\mathbf{x}-\mathbf{x}^{\prime}\right|\right) u\left(\mathbf{x}^{\prime}\right) .
\end{aligned}
$$

The functional $\mathcal{F}$ decreases monotonically in time, possibly reaching a local minimum. For $\epsilon>0$, there is no such $\mathcal{F}$. The necessity of a large-amplitude perturbation for the initiation of nontrivial pattern formation follows directly from the bistability of $F(u)$ and the variational form of the dynamics.

We would like to find an equation of motion for the interface $\mathbf{r}(s)$ in the form $\mathbf{r}_{t}=$ $-\delta \mathcal{F}[\mathbf{r}] / \delta \mathbf{r}$, starting from the equation $u_{t}=-\delta \mathcal{F}[u] / \delta u$. To determine the boundary functional $\mathcal{F}[\mathbf{r}]$ from $\mathcal{F}[u]$ in $(3)$, we restrict ourselves to studying the dynamics of a single island of $u_{\text {in }} \simeq u_{+}$in an infinite sea of $u_{\text {out }} \simeq u_{-}$. We make two assumptions to evaluate the term involving $\nabla u$ : the profile is sharp, so that the gradients are localized on the boundary $\Gamma$, and the curvature of the boundary does not significantly affect the interface profile. The derivation of the contour dynamics using asymptotic expansions will be discussed elsewhere [11]. We proceed here with a heuristic derivation because it yields greater physical insight. The integral of $(\nabla u)^{2}$ is then proportional to the perimeter of the shape, the constant of proportionality being approximately $D\left(u_{\text {in }}-u_{\text {out }}\right)^{2} / l$, with $l$ a characteristic length scale of the profile. One cannot determine $l$ further without specifying the precise form of $F$. 
To evaluate the remaining terms in $\mathcal{F}$, we take $u$ to be piecewise constant. The first term gives $\int F(u)=F\left(u_{\text {in }}\right) \int_{\text {in }}+F\left(u_{\text {out }}\right) \int_{\text {out }}$, where in and out refer to the areas inside and outside of $\Gamma$. The term involving $\mathcal{G}$ can be written as

$$
\begin{aligned}
\iint u \mathcal{G} u= & u_{\text {in }}^{2} \int_{\text {in }} \int_{\text {in }} \mathcal{G}+u_{\text {out }}^{2} \int_{\text {out }} \int_{\text {out }} \mathcal{G} \\
& +2 u_{\text {in }} u_{\text {out }} \int_{\text {in }} \int_{\text {out }} \mathcal{G} .
\end{aligned}
$$

This may be further simplified by an application of Stokes' theorem and the defining relation for $\mathcal{G},\left(\nabla^{2}-\xi^{-2}\right) \mathcal{G}(\mathbf{x})=-\delta(\mathbf{x})$. Apart from an unimportant constant, the energy is then

$$
\mathcal{F}[\mathbf{r}]=\Pi A+\gamma L-\frac{\rho \beta \xi^{2} \Delta^{2}}{2} \oint d s \oint d s^{\prime} \hat{\mathbf{t}} \cdot \hat{\mathbf{t}}^{\prime} \mathcal{G}(R),
$$

where $\gamma \simeq D \Delta^{2} / 2 l$,

$$
\Pi \simeq F\left(u_{\text {in }}\right)-F\left(u_{\text {out }}\right)+\left(\rho \beta \xi^{2} / 2\right)\left(u_{\text {in }}^{2}-u_{\text {out }}^{2}\right),
$$

and $\Delta=u_{\text {in }}-u_{\text {out }}, \mathbf{R}=\mathbf{r}(s)-\mathbf{r}\left(s^{\prime}\right), R=|\mathbf{R}|$. We interpret $\gamma$ as a line tension (associated with the boundary length $L$ ) and $\Pi$ as a pressure (associated with the enclosed area $A$ ). The pressure, which can be of either sign, reflects the difference between the values of the energy $\mathrm{F}(u)$ inside and outside $\Gamma$. Note from the definition of $\Pi$ that the coupling of $u$ to $v$ has created an effective energy $F(u)+\left(\rho \beta \xi^{2} / 2\right) u^{2}$ whose minima determine the values $u_{\text {in }}$ and $u_{\text {out }}$. The nonlocal terms in (5) (and (7), below) have the appearance of a self-induction interaction, although $\mathcal{G}$ provides an exponential screening. While at first sight somewhat unusual, a coupling between tangent vectors appears in other systems with piecewise constant fields [12,13].

Next we determine the equation of motion of $\mathbf{r}$ from that of $u$. This has two parts: (i) relating the time derivatives $u_{t}$ and $\mathbf{r}_{t}$, and (ii) relating the functional derivatives $\delta / \delta u$ and $\delta / \delta \mathbf{r}$. Since $u_{t}$ is nonzero only near the boundary, the approximation $u_{t} \simeq(\Delta / l) \hat{\mathbf{n}} \cdot \mathbf{r}_{t}$ is valid. Likewise, the variation $\delta / \delta u$ is large only near the boundary, suggesting the identification [13] $(\delta / \delta u) \rightarrow(l / \Delta) \hat{\mathbf{n}} \cdot(\delta / \delta \mathbf{r})$. Rescaling time by $(\Delta / l)^{-2}$, the equation of motion is

$$
\hat{\mathbf{n}} \cdot \mathbf{r}_{t}=-\Pi-\gamma \kappa(s)
$$




$$
-\rho \beta \xi^{2} \Delta^{2} \oint d s^{\prime} \hat{\mathbf{R}}\left(s, s^{\prime}\right) \times \hat{\mathbf{t}}\left(s^{\prime}\right) \mathcal{G}^{\prime}(R) .
$$

The prime on $\mathcal{G}$ indicates a derivative with respect to $R$, and the cross product is a scalar in two dimensions.

Note that the repulsion between adjacent fronts depends only on the fact that $\mathcal{G}>0$, and not on the specific form of $\mathcal{G}$ (as can be seen from (5) and the variational form of the equation of motion). This is important, because in a more exact derivation of the contour dynamics that takes the interface profile into account, the function appearing in equation (7) may not be $\mathcal{G}$, but rather one with a less singular behavior as $R \rightarrow 0$ [14. Note however that $\mathcal{G}$ has only a logarithmic, integrable singularity at the origin, so the dynamics derived here is well defined. Even in the presence of such a cutoff in $\mathcal{G}$ the dynamics would not be qualitatively affected, since the sign of $\mathcal{G}$ alone determines whether fronts are attracted or repelled and the large $R$ behavior of the purported function would be unchanged (i.e., exponential decay).

By further redefining the time scale such that the coefficient of the nonlocal term in (7) is unity, one finds only three relevant parameters remain: $\xi$ and a rescaled $\gamma$ and $\Pi$. The model (7) was investiged numerically with a pseudospectral technique. Figure 3 shows the evolution of a circle seeded with sinusoidal perturbations. A complicated labyrinth forms due to the repeated fingering of the boundary. The interface never crosses itself, due to the repulsion between the adjacent fingers. The competition between the inward pressure $\Pi$ and the repulsion within a finger sets the finger width, while the interfinger distance is set by the inhibitor length scale $\xi$ alone. The nonlocal nature of the dynamics severely limits the time scale over which the evolution may be followed numerically; with further computation, the pattern in Fig. 3 would continue to evolve beyond the final picture.

Figure 6 shows the evolution of an island with a larger value of $\Pi$ than that in Fig. [3, implying a larger energy difference between the two possible homogeneous states of $u$. The area enclosed by the interface changes dramatically over time, the pattern simply shrinking to a circle. This shrinkage of a fingered structure has also been seen in the work of Lee, et 
al. [6].

To gain insight into the basic mechanism of the fingering instability, we develop an approximate local dynamics valid when $\xi \kappa \ll 1$ and the shape is approximately circular [15]. Observe that the most important contribution to the double integral in (5) is from the region $\left|s^{\prime}-s\right| \leq \xi$. Near the point $\mathbf{r}(s)$, expand the scalar product as $\hat{\mathbf{t}}(s) \cdot \hat{\mathbf{t}}\left(s^{\prime}\right) \simeq$ $1-(1 / 2)\left(s-s^{\prime}\right)^{2} \kappa(s)^{2}+\cdots$, then perform the $s^{\prime}$ integral over the extended region $[-\infty, \infty]$ to obtain an effective local energy functional $\mathcal{F}_{v}$ :

$$
\mathcal{F}_{v} \simeq \oint d s\left\{\gamma_{v}+\frac{1}{2} k_{c}(\xi \kappa)^{2}+\mathcal{O}\left((\xi \kappa)^{4}\right)\right\}
$$

The first term contributes to an effective line tension, while the second we recognize from elasticity theory as the bending energy of a rigid rod. A detailed calculation yields $\gamma_{v}=$ $-\rho \xi^{3} / 4$ and $k_{c}=\rho \xi^{3} / 8$. While $k_{c}$ is positive, $\gamma_{v}$ is negative. The effective line tension $\tilde{\gamma}=\gamma+\gamma_{v}$ can be negative, favoring proliferation of the interface. Again, note that the sign of $\gamma_{v}$ is opposite that of $\mathcal{G}$, and hence the physics of fingering depends only on the sign of $\mathcal{G}$ and not its specific functional form.

Under the local approximation, the interface motion is

$$
\hat{\mathbf{n}} \cdot \mathbf{r}_{t} \simeq-\Pi-\tilde{\gamma} \kappa-k_{c} \xi^{2}\left(\kappa_{s s}+\frac{1}{2} \kappa^{3}\right)
$$

similar to "geometric" models [16] of crystal growth. In a linear stability analysis of a circular shape, the growth rate $\sigma_{n}$ of the $n^{\text {th }}$ mode is $\sigma_{n} \sim-\tilde{\gamma} n^{2}-k_{c} \xi^{2} n^{4}$. For $\tilde{\gamma}<0$, there is a band of unstable modes whose maximum extent is limited by the rigidity. In the numerical studies shown here, the effective tension is negative in both cases. In Fig. 3, the inward pressure is insufficient to prevent the proliferation of the interface, while in Fig. 4 the larger pressure overcomes the negative surface tension.

In summary, starting from a reaction-diffusion system in two dimensions, we have constructed a boundary dynamics useful in describing pattern formation. Possible extensions include studying inertial effects from higher order terms in $\epsilon$, and the application of these methods to higher-dimensional problems. It is also of interest to determine if the particular chemical kinetics in the experiments of Lee, et al. may indeed be mapped onto the 
interface model developed here. Finally, it is possible to extend these methods to study the more physically relevant slow-inhibitor problem [1]. Perhaps issues such as spiral wave stability [8,17] could be given a more intuitive interpretation when studied from a geometric viewpoint.

We are grateful to K.J. Lee, W.D. McCormick, Q. Ouyang, and H.L. Swinney for communication of their results prior to publication and for kindly providing Fig. 1, and thank V. Hakim and S. Leibler for important discussions on localized states, and D. Barkley, S. Erramilli, M.J. Shelley, and A.T. Winfree for discussions and correspondence. This material is based upon work supported under an N.S.F. Graduate Fellowship (DMP), N.S.F. Presidential Faculty Fellowship Grant DMR-9350227 and the Alfred P. Sloan Foundation (REG). 


\section{REFERENCES}

[1] A.M. Turing, Philos. Trans. R. Soc. London Ser. B 237, 37 (1952).

[2] Q. Ouyang and H.L. Swinney, Nature 352, 610 (1991).

[3] S. Koga and Y. Kuramoto, Prog. Theor. Phys. 63, 106 (1980).

[4] T. Ohta, M. Mimura, and R. Kobayashi, Physica D 34, 115 (1989).

[5] M. Seul, L.R. Monar, L. O’Gorman, and R. Wolfe, Science 254, 1616 (1991); R.E. Rosensweig, Ferrohydrodynamics (Cambridge University Press, Cambridge, 1985; A.J. Dickstein, S. Erramilli, R.E. Goldstein, D.P. Jackson, and S.A. Langer, Science 261, 1012 (1993).

[6] K.J. Lee, W.D. McCormick, Q. Ouyang, and H.L. Swinney, Science 261, 189 (1993).

[7] For direct numerical simulations of the related Gray-Scott model, see J.E. Pearson, Science 261, 192 (1993). See also A. Hagberg and E. Meron, "Domain Walls in Non-Equilibrium Systems and the Emergence of Persistent Patterns," preprint (1993).

[8] J.P. Keener, SIAM J. Appl. Math. 46, 1039 (1986); A.T. Winfree, SIAM Review 32, 1 (1990); E. Meron, Phys. Reports 218, 1 (1992), and references therein.

[9] H. FitzHugh, in Biological Engineering, ed. H.P. Schwan (McGraw-Hill, New York, 1969).

[10] See, e.g. P.C. Fife, Dynamics of Internal Layers and Diffusive Interfaces (SIAM, Philadelphia, 1988), and references therein.

[11] D.M. Petrich, R.E. Goldstein, and D. Muraki, unpublished.

[12] D.G. Dritschel, J. Fluid Mech. 172, 157 (1986); S.A. Langer, R.E. Goldstein, and D.P. Jackson, Phys. Rev. A 46, 4894 (1992).

[13] R.E. Goldstein and D.M. Petrich, Phys. Rev. Lett. 69, 555 (1992).

[14] We thank an anonymous referee for emphasizing this point. 
[15] B. Duplantier, R.E. Goldstein, V. Romero-Rochín, and A.I. Pesci, Phys. Rev. Lett. 65, 508 (1990).

[16] R.C. Brower, D.A. Kessler, J. Koplik, and H. Levine, Phys. Rev. A 29, 1335 (1984).

[17] D. Barkley, Phys. Rev. Lett. 68, 2090 (1992); See also Ref. [8]. 


\section{FIGURES}

FIG. 1. Pattern formation in the chemical system of Lee, McCormick, Ouyang, and Swinney [6]. White and black regions correspond, respectively, to low and high $\mathrm{pH}$, made visible with a $\mathrm{pH}$ indicator. Figure courtesy of Lee, et al. [6].

FIG. 2. Space-time portrait of the interaction of two fronts, from numerical solution of Eq. 1 with $\epsilon=0.008, D=0.2, \rho=0.1, \alpha=\beta=0.2, F^{\prime}(u)=a u+b u^{2}+d u^{3}$, with $a=0.23, b=-1.23$, and $c=1.0$. Solid lines show $u(x, t)$, dashed lines are $v(x, t)$, with time increasing upward.

FIG. 3. Numerical solution of (7) with $\gamma=2.0, \Pi=0.02$, and $\xi=1.0$. The initial condition is a perturbed circle of radius 15 , and the time interval between shapes is 12 .

FIG. 4. Shrinkage of a domain with $\gamma=2.0, \Pi=2.0$, and $\xi=1.0$. The time interval between shapes is 1 . 